\title{
Avaliação do grau de dependência nas atividades de vida diária em idosos da cidade de Fortaleza - Ceará
}

\author{
Level of independence in activities of daily life in low-income elderly from Fortaleza-Ceará \\ Evaluation of dependence degree in daily activities of life in elder of Fortaleza - Ceará
}

\begin{abstract}
Maria Josefina da Silva ${ }^{1}$, Marcos Venicios de Oliveira Lopes, Maria Fátima Maciel Araújo ${ }^{3}$, Geńdice Loma Andrade de Moraes $^{4}$
\end{abstract}

\begin{abstract}
RESUMO
Objetivo: a finalidade deste estudo foi avaliar o grau de dependência de idosos de baixa renda para as Atividades de vida diária (AVDs). Métodos: estudo transversal, junto a 385 idosos residentes em bairros periféricos da cidade de Fortaleza - CE, caracterizados como pessoas pobres, com baixa ou nenhuma escolaridade, do sexo feminino e viúvas. O instrumento consistiu de formulário contendo 11 AVDs com gradientes de dependência "sem restrição"(0), "com restrição - pouca (1) e muita (2)" e "não consegue"(3). Cada AVD continha três variações de complexidade para sua realização. Resultados: os resultados revelaram que as atividades mais comprometidas foram "alimentação e hidratação", "locomoção", "atividades realizadas na casa" em especial as consideradas pesadas, "lazer e recreação". Conclusão: conclui-se que há um forte comprometimento da qualidade de vida dos idosos frente às restrições identificadas e aos parcos recursos institucionais, comunitários e familiares que dispõem para compensar as dependências individuais identificadas.
\end{abstract}

Descritores: Envelhecimento; Avaliação geriátrica; Atividades cotidianas

\section{ABSTRACT}

Objective: to evaluate the level of independence in activities of daily living among low-income elderly Methods: this was a cross-sectional study. The sample consisted of 385 low-income elderly residents of Fortaleza. Inclusion criteria were low income, low educational level, female, and widows. Data were collected using an instrument that measured 11 activities fo daily living. Each of the activities was scored on a scale from 'no restriction" (0) to 'cannot do' (3). Results: the analysis showed that the major activities impacting the level of independence were "ambulation", "household chores", and "leisure and recreation". Conclusions: there was a significant decrease in quality of life of elderly because of a decrease in the level of independence in performing activities of daily living. In addition, there was a lack of instituional resources, and community and family support for this sample of low income elderly.

Keywords: Aging; Geriatric assessment; Activities of daily living

\section{RESUMEN}

Objetivo: la finalidad de este estudio fue evaluar el grado de dependecia de ancianos de bajo recursos para las Actividades de vida diaria (AVDs). Método: se trata de un estudio tranversal, dessarrollado con 385 ancianos residentes en barrios periféricos de la ciudad de Fortaleza - CE, caracterizados como personas pobres, con baja o ninguna escolaridad, viudas, del sexo femenino. El instrumento consistió de un formulario que contenía 11 AVDs con grados de dependencia "sin restricción" (0), "con restricción - poca (1) y mucha (2)" y "no consigue"(3). Cada AVD contenía tres variaciones de complejidad para su realización. Resultados: los resultados revelaron que las actividades más comprometidas fueron "alimentación e hidratación", "locomoción", "actividades realizadas en la casa" en especial las consideradas pesadas, "Ocio y recreación". Conclusión: se concluye que hay un fuerte compromiso de la calidad de vida de los ancianos frente a las restricciones identificadas y a los escasos recursos institucionales, comunitarios y familiares que disponen para compensar las dependencias individuales identificadas.

Descriptors: Evejecimento; Evaluación geriátrica; Actividades cotidianas

\footnotetext{
${ }^{1}$ Doutora em Enfermagem; Coordenadora do Projeto "O Idoso dependente e o universo do Cuidado Domiciliar"; Docente do Departamento de Enfermagem, Universidade Federal do Ceará - UFC - Fortaleza (CE), Brasil.

${ }^{2}$ Doutor em Enfermagem, Docente do Departamento de Enfermagem, Universidade Federal do Ceará - UFC - Fortaleza (CE), Brasil.

${ }^{3}$ Doutor em Enfermagem, Docente do Departamento de Enfermagem, Universidade Federal do Ceará - UFC - Fortaleza (CE), Brasil.

${ }^{4}$ Mestranda em Enfermagem/DENF/UFC. Enfermeira do Programa de Saúde da Família de Fortaleza (CE), Brasil.
} 


\section{INTRODUÇÃO}

O envelhecimento populacional é um evento inédito na história humana. $\mathrm{O}$ contingente de pessoas consideradas idosas, que no Brasil é definido como as pessoas acima de 60 anos $^{(1)}$, aumenta com uma velocidade acima de todas as outras faixas etárias, e em nosso país, já constitui $8,9 \%$ da população total ${ }^{(2)}$.

Na proporção que a longevidade aumenta, a dependência tende a aumentar em decorrência de múltiplos fatores ${ }^{(3)}$, embora não possa ser estabelecida uma relação direta de causa e efeito. As alterações orgânicas, funcionais e psicológicas decorrentes do envelhecimento normal ${ }^{(4)}$ são variadas e dependem de como cada um se preparou para esta etapa da vida, de sua capacidade física, da manutenção das atividades que proporcionem prazer e desenvolvimento intelectual e pessoal e da rede social que contribuem para a manutenção de sua autonomia ${ }^{(5)}$. Na verdade, não é o avanço da idade que marca as etapas mais significativas da vida, e sim um processo contínuo de reconstrução( ${ }^{(6)}$.

Autonomia e dependência são aspectos que demarcam ser velho (dependente) ou idoso (autônomo), conforme concepção de idosos durante as consultas de enfermagem em unidades de saúde, visitas domiciliárias e em grupos de convivência dos quais os autores participam como coordenadores ou animadores. Para estes, só é velho quem perde a capacidade de administrar seu próprio cotidiano ${ }^{(7-9)}$.

A dependência, por si só, não constitui um evento negativo. Em diferentes etapas da vida a pessoa pode ser ou não dependente, temporária ou definitivamente ${ }^{(10)}$. A dependência se configura mais relevante quando esta surge em decorrência de eventos ocorridos na etapa final da vida, e as atividades cotidianas são afetadas por esta dependência.

Destaca-se que a mulher é mais afetada na sua autonomia para as Atividades de vida diária (AVD), especialmente em idades mais avançadas ( $>85$ anos) por conseqüências de agravos prolongados e progressivamente incapacitantes ${ }^{(11)}$. Nosso objetivo foi, portanto, avaliar o grau de dependência dos idosos de baixa renda para as AVD. Este estudo é parte do Projeto "O Idoso dependente e o universo do cuidado domiciliar", financiado pela Fundação Cearense de Apoio ao Desenvolvimento Científico e Tecnológico - FUNCAP.

\section{MÉTODOS}

Estudo quantitativo do tipo transversal de caráter exploratório e descritivo. Desenvolvido junto a idosos residentes em bairros periféricos da cidade de Fortaleza Ceará / Brasil. A população estudada foi composta pelos idosos dependentes, considerados como tais os que necessitavam de cuidador, familiar ou não e identificados ou cadastrados pelas equipes de saúde da família, e residentes nas áreas de abrangência das unidades Básicas de Saúde da Familia nos bairros do Pirambu, Antonio Bezerra e Dom
Lustosa. Para cálculo do tamanho da amostra foi utilizada uma fórmula desenvolvida para estudos de populações infinitas, e que leva em consideração o intervalo de confiança e o erro amostral. A fórmula utilizada foi a seguinte:

$$
\mathrm{n}=\left\{\frac{\mathrm{Za}(0,5)}{\mathrm{E}}\right\} \begin{aligned}
& \mathrm{Za}=\text { Nivel de confiança com contagens } \mathrm{Z} \\
& \mathrm{E}=\text { erro aleatório } \\
& \mathrm{N}=\text { Amostra }
\end{aligned}
$$

A partir desta fórmula foi delimitado um nível de confiança de $95 \%\left(Z_{a}=1,96\right)$, um erro amostral de 5\% (E $=0,05)$. A amostra calculada foi arredondada e estabelecida em 385 indivíduos. Considerou-se, neste estudo, a margem de erro comumente utilizada em estudos transversais conforme literatura consultada ${ }^{(12-13)}$.

Para a coleta de dados foi utilizada uma escala de graus de dependência para as atividades da vida diária, baseada no modelo de vida ${ }^{(10,14)}$ com 11 itens de avaliação: locomoção; alimentação e hidratação; eliminação; vestir-se; atividades de casa; sono e repouso; lazer e recreação; sexualidade; comunicação e uso de transportes construída pelos autores. O instrumento foi avaliado por enfermeiros do PSF da área do estudo e realizado um pré-teste. A elaboração do instrumento ancorado nas atividades da vida prende-se ao fato de que é o modelo adotado para a prática do cuidado de enfermagem à criança, à gestante e ao idoso, atendidos no Centro de Desenvolvimento Familiar, unidade de atenção básica da UFC. Para cada atividade foram detalhados três subitens com grau de dificuldade crescente. Exemplo: na atividade locomoção: foram discriminados: em todos os ambientes da casa; em áreas próximas e para qualquer lugar da cidade. A dependência foi avaliada, considerando: sem restrição; com pouca restrição; com muita restrição e não consegue. Foi construída, pelos autores, uma planilha com a interpretação de cada um dos graus de dependência, de modo a padronizar a coleta de dados efetuada por agentes comunitários de saúde (ACS) que trabalham nas Unidades Básicas de Saúde da Família localizadas nos bairros onde o estudo foi realizado. O instrumento foi considerado pelos agentes comunitários como de fácil compreensão e aplicação.

A coleta de dados foi realizada durante visita domiciliária realizada pelos agentes comunitários de saúde que atuam nas áreas selecionadas. Todos os agentes (45) foram treinados pelos pesquisadores. A escolha dos ACS deveu-se a estes terem a informação dos idosos que tinham algum nível de dependência caracterizado pela necessidade de um cuidador domiciliar em, no mínimo, três atividades da vida, segundo o modelo ${ }^{(10,14)}$. O período da coleta de dados foi de setembro a janeiro de 2004.

Foram seguidos todos os procedimentos referentes à pesquisa em seres humanos conforme a Resolução n. ${ }^{\circ}$ 196/ 96 do Conselho Nacional de Saúde ${ }^{(15)}$.

Para a análise dos dados foram geradas estatísticas com 
a freqüência absoluta, percentuais e intervalos de confiança de $95 \%$ de cada uma das variáveis. Em seguida, foram aplicados testes de qui-quadrado para verificação de associação entre as variáveis. Para organização final e apresentação dos resultados no presente artigo, optou-se pela descrição dos achados. A análise apresentada foi baseada na leitura das estatísticas descritivas, bem como, na análise dos valores p encontrados. Para significância estatística adotouse um nível de $5 \%(a=0,05)$.

\section{RESULTADOS}

Dos idosos avaliados $68,6 \%$ eram do sexo feminino; $78,4 \%$ eram analfabetos ou apenas alfabetizados; $80,1 \%$ eram católicos; $48,9 \%$ eram viúvos e $37,0 \%$ casados ou em união consensual: $66,2 \%$ recebiam entre 1 e 2 salários mínimos $(\mathrm{R} \$ 240,00)$.

Apresentaremos apenas os dados relativos às atividades da vida cujos resultados foram significantes considerando os objetivos do trabalho.

$\mathrm{Na}$ avaliação do grau de dependência, o item Locomoção, foi avaliado nos idosos considerando a capacidade dos mesmos se deslocarem dentro e fora do ambiente domiciliar. No interior do ambiente doméstico, 19,24\% dos idosos apresentaram restrição total; para áreas próximas ao domicilio 40,1\%; para qualquer lugar da cidade $65 \%$ dos idosos com restrição total. Foi encontrada associação estatisticamente significante entre o tipo de atividade de locomoção, dicotomizada em "dentro" e "fora" do domicílio, e o grau de dependência observado nos idosos, dicotomizado em "com" e "sem" dependência (valor p < 0,00001).

Quanto à alimentação e bidratação os idosos que apresentaram restrição total foram $42,9 \%$. Para alimentar-se $18,8 \%$ foram avaliados com restrição total. No preparo dos alimentos $63,9 \%$ apresentaram restrição total. Ao se dicotomizar a variável alimentação em atividades da vida diária (alimentarse) e atividades instrumentais (adquirir e preparar), encontrouse associação estatisticamente significante com o grau de dependência $(p<0,00001)$, indicando comprometimento maior das atividades instrumentais.

$\mathrm{Na}$ avaliação da eliminação, o controle vesical e anal diurno estava totalmente restrito em $12,2 \%$ dos idosos. No controle vesical e anal noturno, 15,0\% apresentavam restrição total. Foi encontrada associação estatisticamente significante entre o controle esfincteriano e o grau de dependência, indicando maior acometimento do controle no período noturno $(\mathrm{p}=$ 0,0002).

No item higiene corporal, a capacidade de banhar-se se apresentou com restrição total em 23,2\%. A atividade de cortar as unhas dos pés não era desenvolvida por $72,2 \%$ dos idosos. Por fim, a higiene oral esteve totalmente restrita em 20,5\%. Associação estatisticamente significante foi encontrada entre a capacidade de higienizar-se e o grau de dependência do idoso, indicando comprometimento mais acentuado da atividade de cortar as unhas.

Em relação às atividades desenvolvidas em casa, para as atividades leves 38,7\% dos idosos apresentaram total restrição. Nas atividades médias, essa proporção aumentou para 39,3\%. Para as atividades pesadas, $89,5 \%$ apresentaram restrição total. Foi encontrada associação estatisticamente significante entre o tipo de atividade desenvolvida e o grau de dependência do idoso ( $p<0,00001)$, indicando acometimento heterogêneo desta capacidade, particularmente para atividades consideradas pesadas.

$\mathrm{Na}$ avaliação do sono e repouso, a restrição total foi identificada em $14,7 \%$ restrição total. O sono noturno estava totalmente afetado em 16,6\% dos idosos. 29,4\% faziam uso permanente de medicamentos para dormir. Foi detectada associação estatisticamente significante entre o comprometimento do sono e repouso, com maior destaque para o uso de medicamentos para dormir, e o grau de dependência apresentado pelos idosos ( $\mathrm{p}<0,00001)$.

No item lazer e recreação, $72 \%$ dos idosos não participavam de grupos de convivência. No domicílio, o lazer estava totalmente restrito em $40,1 \%$. Os passeios eram totalmente restritos em $64,8 \%$ dos idosos. Associação estatisticamente significante foi encontrada entre a restrição ao lazer e a recreação e o grau de dependência dos idosos ( $p<0,000001)$. Neste caso a heterogeneidade se deu pelo maior percentual de não restrição para o lazer no domicílio.

A capacidade de comunicação apresentou os seguintes resultados: na compreensão cognitiva, 17,5\% apresentavam restrição total; a compreensão visual estava restrita totalmente em 30,5\%; para a identificação de sons 13,9\% apresentavam restrição total; na verbalização de suas necessidades a restrição total foi identificada em $12,4 \%$ dos idosos. Foi encontrada associação estatisticamente significante entre o tipo de atividade de comunicação e o grau de dependência $(\mathrm{p}<0,000001)$, mostrando acometimento heterogêneo desta capacidade com destaque para a compreensão visual.

O último ponto avaliado foi o uso de transportes, onde a atividade de subir e descer dos mesmos esteve totalmente restrita em 65,6\%. O uso de transportes urbanos esteve totalmente restrito em 70,2\%. Para transportes interurbanos encontramos $70,9 \%$ dos idosos com restrição total. Foi detectada associação estatisticamente significante entre o tipo de atividade para uso de transportes e o grau de dependência $(\mathrm{p}=0,022)$ com comprometimento relacionado à distância a ser percorrida.

\section{DISCUSSÃO}

A capacidade de preservação da independência, aqui entendida como a capacidade funcional, em sua expressão máxima, significa poder sobreviver sem ajuda para as atividades instrumentais da vida diária e de autocuidado. Dependência é definida como a incapacidade de a pessoa 
funcionar satisfatoriamente sem ajuda, quer devido a limitações físico-funcionais, quer devido a limitações cognitivas $^{(16)}$. Observa-se uma diminuição acelerada do tamanho da família, que, segundo o último senso é 3,4 pessoas para o Brasil ${ }^{(17)}$ e 3,78 pessoas para o Nordeste, onde nosso estudo se situa. Este dado indica dificuldades da permanência do idoso nos domicílios, especialmente os que têm graus de dependência aumentados chegando, por vezes, a um nível de quase totalidade agravado pela falta de um cuidador. O contexto sócio-cultural-econômico pelo qual o país está passando, dificulta a busca de soluções para a manutenção do idoso em seu ambiente, quando a dependência passa a se tornar um problema familiar, dada a demanda maior por cuidados e serviços de saúde, pela complexidade do cuidado no interior dos lares.

Os idosos participantes do estudo são pessoas pobres, com baixa ou nenhuma escolaridade, a maioria do sexo feminino e viúvas. Este é o perfil da velhice feminizada ${ }^{(18-19)}$. Embora não tenha sido, neste estudo, realizada uma estratificação entre sexos, observa-se na literatura que as mulheres idosas são, em sua maioria viúvas ${ }^{(18)}$, empobrecidas, com baixa escolaridade e com uma carga de doenças ou agravos maior que os seus pares masculinos.

$\mathrm{Na}$ análise das atividades de vida diária, segundo o modelo adotado no estudo ${ }^{13}$ concentramos nossa atenção para a restrição total, ou seja, a incapacidade de realizar as atividades.

$\mathrm{Na}$ atividade de locomoção, temos 19,2\% incapazes de se locomover em casa. Isto indica que o idoso nesta situação acaba sendo dependente nas demais atividades. Estudo longitudinal demonstra que a perda de mobilidade e inatividade física podem ampliar em duas vezes o risco de morte ${ }^{(20)}$. A falta de mobilidade dos idosos estudados acaba por tirar deste a capacidade de gerenciar sua vida, sua autonomia. A mobilidade é, geralmente, a primeira AV comprometida e, se considerarmos que esta é necessária para a maioria das AV, isso resultará na perda da independência $^{(10)}$.

No ambiente familiar nossos entrevistados não dispõem de equipamentos para compensar a dificuldade de deambulação como andadores, muletas, bengalas, cadeiras de rodas nem de serviços de reabilitação. Suas residências também não oferecem condições de deambulação sem o risco de quedas e há limitação de trânsito, pelos espaços pequenos. Assim, observamos que 65\% dos idosos não saem de casa sozinhos, limitando suas vidas ao ambiente doméstico, por não disporem de cuidadores que os levem a algum lugar de seu interesse. A dependência para a deambulação pode ser causada pela falta de atividade, mudanças de hábitos, consumo de drogas psicoativas, consumo de medicamentos, situações de enfermidades, preocupações e tristezas ${ }^{(21)}$.

Este aspecto compromete o lazer e, neste item, observamos que a grande maioria $(42 \%$ e $64,8 \%)$ não freqüenta grupos de convivência e nem realiza passeios. Percebemos a importância do lazer para a preservação da satisfação em viver e qualidade de vida e a literatura confirma esta observação ${ }^{(22)}$.

Também ligado a esta atividade de locomoção, relacionamos ao uso de transportes, que segue a mesma tendência, com mais de $60 \%$ dos entrevistados com restrição total para subir ou descer $(65,5 \%)$, usar transportes urbanos $(70,0 \%)$ e interurbanos $(70,9 \%)$.

O recebimento da aposentadoria, na maioria das vezes em unidades bancárias distantes do domicílio, com necessidade de atravessar vias de alta velocidade ou o uso de transportes inadequados ao idoso, acaba por tornar esta tarefa inviável. O idoso se vê obrigado a transferir esta atividade para um parente o que, freqüentemente, retira dele a autonomia financeira, pois se torna irreversível o processo. Embora aparentemente irrelevante, a perda do controle de seus recursos leva o idoso a perceber como é limitado seu papel familiar dada exclusão a que ele é submetido. Não é raro ouvirmos queixas de idosos que perderam completamente sua capacidade de administrar seus recursos, com a conseqüente perda da capacidade de atender suas necessidades, até de compra de medicamentos que lhes são essenciais.

As atividades relacionadas ao autocuidado, como a higiene pessoal e vestir-se ficaram comprometidas para um número significativo de idosos entrevistados. A dependência total nesta atividade tem repercussão mais profunda que simplesmente depender de um cuidador. O pudor que a grande maioria dos idosos preserva quanto a exposição do corpo, mesmo que para pessoas de sua intimidade, cria constrangimento que pode levar à complicação de sua saúde. A demora para que o idoso aceite que outro faça sua higiene íntima propicia o aparecimento de úlceras de pressão, de infecções genitais e outros agravos.

A necessidade de um cuidador, quando a dependência é total, é problematizada uma vez que, como relata a literatura ${ }^{(21,23)}$, recai sobre a mulher, filha ou nora, já próximas da meia idade ou velhice. Por outro lado, o cuidador, muitas vezes, abdica de itens importantes para sua vida pessoal como trabalho, lazer, casamento e outros. Esta é uma realidade que não pode ser ignorada nas famílias de baixa renda.

Chama-nos a atenção os idosos que fazem uso permanente de medicamentos para dormir (29,4\%). A dificuldade de conciliar o sono é uma queixa freqüente em idoso e é esperada uma mudança do padrão de sono. Este problema se torna mais complexo quando a residência do idoso é dividida com pessoas que têm hábitos de sono e vigília diferentes dos seus ou seu entorno é ruidoso. Nas áreas mais pobres da cidade de Fortaleza, é freqüente que as pessoas transformem suas casas em bares que ficam abertos até tarde da noite e essa tem sido uma queixa comum, levando, por vezes, à mudança de endereço pelo 
idoso, trazendo-lhe prejuízo monetário e de rede social de apoio.

Por fim, cabe-nos analisar a atividade de comunicação com destaque para a dificuldade de comunicação visual. Esta limitação também contribui para a limitação na locomoção. Nas áreas estudadas, é observável a falta de espaços para o trânsito de idoso sem o risco de atropelamento, de quedas de calçadas irregulares e de entulhos depositados nas áreas residenciais. Não há uma estrutura urbana que viabilize ao idoso com déficit visual ter autonomia em ambientes circundantes de sua residência.

\section{CONCLUSÃO}

Observamos, a partir dos dados revelados na pesquisa, a necessidade dos serviços de saúde e dos profissionais que trabalham com famílias de idosos dependentes avaliarem o grau de comprometimento para envidarem esforços no sentido de auxiliar estas famílias, e ao próprio idoso, maximizar sua capacidade de tocar seu cotidiano utilizando toda sua capacidade funcional.

A dependência manifestada pelos idosos abarcou todas as áreas das atividades de vida diária. Em sua maioria, tais atividades estavam comprometidas num foco específico, entretanto foi possível identificar atividades com total comprometimento. A dependência era maior quanto mais complexa era a atividade, e a necessidade de um cuidador foi patente.

Parcerias entre serviços, profissionais, famílias e idosos e sociedade em geral focando estes aspectos contribuem, de forma indelével, para preservação da auto-estima do ser idoso e aceitação do mesmo no seu contexto significativo.

\section{REFERÊNCIAS}

1. Equipe RT. Estatuto do Idoso e legislação correlata. São Paulo: Editora Revista dos Tribunais; 2004.

2. IBGE. Pesquisa Nacional de Amostragem Domiciliar 2001 [sítio na Internet]. Brasília:IBGE; 2003. [citado 2004 Abr 8] Disponível em: http://www.ibge.gov.br/home/estatistica/ população/censo2000/população/pop-censo2000

3. Pavarini SCI, Néri AL. Compreendendo dependência, independência e autonomia no contexto domiciliar: conceitos, atitudes, comportamento. In: Duarte YAO, Diogo MJD. Atendimento domiciliar: um enfoque gerontológico. São Paulo: Atheneu;2000. p.7-10.

4. Papaléo Netto M. O estudo da velhice no século XX: histórico, definição de campo e termos básicos. In: Freitas EV, Py L, Néri AL, Cançado FAX, Gorzoni ML, Rocha SM. Tratado de geriatria e gerontologia. Rio de Janeiro: Guanabra Koogan; 2002. p. 2-12.

5. Coppard L. La autoatención de la salud y los ancianos. In: Organización Panamericana de la Salud. Hacia el bienestar de los ancianos. Washington (DC); 1985. p.45-58. (OPSPublicación Científica, 492).

6. Debert GG. A reinvenção da velhice: socialização e processos de reprivatização do envelhecimento. São Paulo: EDUSP; 1991.

7. Pires ZRS, Silva MJ. Autonomia e capacidade decisória dos idosos de baixa renda: uma problemática a ser considerada na saúde do idoso. Rev Eletrônica Enferm [periódico na Internet]. 2001 [citado 2003 Jun 12]; 3(2) [cerca de 5 p.]. Disponível em : http://www.fen.ufg.br/Revista/revista3_2/autonomia.html

8. Barbosa NS, Silva MJ, Miranda MDIC. O enfrentamento das dificuldades de vida cotidiana pelos idosos de baixa renda. Rev RENE. 2002; 3(1): 14-20.

9. Ramos LR. Fatores determinantes do envelhecimento saudável em idosos residentes em centro urbano: Projeto Epidoso, São Paulo. Cad Saúde Pública = Rep Public Health. 2003; 19(3): 793-8.

10. Roper N, Logan W, Tierney AJ. O modelo de enfermagem: baseado nas atividades de vida diária. Lisboa: Climepsi; 2001.

11. von Strauss E, Agüero-Torres H, Kareholt I, Winblad B, Fratiglioni L. Women are more disabled in basic activities of daily living than men only in very advanced ages: a study on disability, morbidity, and mortality from the Kungsholmen Project. J Clin Epidemiol. 2003; 56(7):669-77.

12. Rea LM, Parker RA. Metodologia de pesquisa: do planejamento à execução. São Paulo: Pioneira; 2002.

13. Jekel JF, Elmore JG, Katz DL. Epidemiologia, bioestatística e medicina preventiva. Porto Alegre: Artmed; 2002.

14. Diogo MJDE. Consulta de Enfermagem. In: Papaléo Netto M. Gerontologia. São Paulo: Atheneu; 1996. p.209-21.

15. Brasil. Conselho Nacional de Saúde. Resolução no. 196/96. Decreto ${ }^{\circ}$. 93.933 de 14 de janeiro de 1987. Estabelece diretrizes e normas regulamentadoras de pesquisas envolvendo seres humanos. Brasil: CNS; 1996.

16. Neri AL. Palavras-chave em gerontologia. Campinas: Alínea; 2001.

17. IBGE. Pesquisa Nacional por amostra de domicílios 1999. Microdados [CD-ROM]. Rio de Janeiro: IBGE; 2000.

18. Hayflick L. Como e porque envelhecemos. Rio de Janeiro: Campus; 1997.

19. Walsh F. A Família no estágio tardio de vida. In: Carter B, McGoldrick M. As mudanças no ciclo de vida familiar: uma estrutura para a terapia familiar. 2a. ed. Porto Alegre: Artes Médicas; 1995 . p. 269-87.

20. Hirvensalo M, Rantanen T, Heikkinen E. Mobility difficulties and physical activity as predictors of mortality and loss of independence in the community-living older population. J Am Geriatr Soc. 2000; 48(5):493-8.

21. Trocóniz MIF, Cerrato IM, Veiga PD. Cuando las personas mayores necesitan ayuda. Guia para cuidadores y familiares. Resolviendo problemas difíciles. Madrid: Ministério de Trabajo y asuntos sociales. SGAS; IMSERSO, 2002. v. 2, p. 22-8.

22. Coelho Neto A, Araújo ALC. As dimensões da vida. Fortaleza: ABC Fortaleza; 1998.

23. Silva LF, Guedes MVC, Moreira RP, Souza ACC. Doença crônica: o enfrentamento pela família. Acta Paul Enfermagem. 2002;15(1):40-7. 
Tabela 1- Estatísticas da escala de avaliação de grau de dependência nas atividades da vida diária de idosos. Fortaleza, 2004.

\begin{tabular}{|c|c|c|c|c|}
\hline Atividade da vida diánia & Média & Mediana & Desvio - Padrão & Valorp \\
\hline \multicolumn{5}{|l|}{ 1. Locomoção } \\
\hline Em todos os ambientes da casa & 1,17 & 1,00 & 1,155 & \multirow[t]{3}{*}{0,0000} \\
\hline Para áreas próximas da casa & 1,93 & 2,00 & 1,098 & \\
\hline Para qualquer lugar da cidade & 2,50 & 3,00 & 0,819 & \\
\hline \multicolumn{5}{|l|}{ 2. Alimentação e hidratação } \\
\hline Aquisição de alimentos & 1,62 & 2,00 & 1,329 & \multirow[t]{3}{*}{0,0000} \\
\hline Alimentação & 0,91 & 0,00 & 1,202 & \\
\hline Preparo de alimentos & 2,16 & 3,00 & 1,200 & \\
\hline \multicolumn{5}{|l|}{ 3. Eliminação: uninária e fecal } \\
\hline Controle do esfíncter vesical e anal diurno & 0,83 & 0,00 & 1,065 & \multirow[t]{2}{*}{0,0002} \\
\hline Controle do esfíncter vesical e anal noturno & 1,04 & 1,00 & 1,074 & \\
\hline \multicolumn{5}{|l|}{ 4. Higiene corporal } \\
\hline Banho & 1,03 & 0,00 & 1,250 & \multirow[t]{3}{*}{0,0000} \\
\hline Corte de unhas & 2,33 & 3,00 & 1,151 & \\
\hline Higiene oral & 0,91 & 0,00 & 1,222 & \\
\hline \multicolumn{5}{|l|}{ 5. Vestir-se } \\
\hline Calça sapatos e chinelos & 1,54 & 2,00 & 1,328 & \multirow[t]{3}{*}{0,0800} \\
\hline Retira roupas de armários e gavetas & 1,46 & 1,00 & 1,356 & \\
\hline Veste todas & 1,27 & 1,00 & 1,304 & \\
\hline \multicolumn{5}{|l|}{ 6. Atividades realizadas em casa } \\
\hline Atividades leves & 2,79 & 3,00 & 0,670 & \multirow[t]{3}{*}{0,0000} \\
\hline Atividades médias & 2,00 & 3,00 & 1,283 & \\
\hline Atividades pesadas & 2,66 & 3,00 & 0,782 & \\
\hline \multicolumn{5}{|l|}{ 7. Sono e repouso } \\
\hline Sesta durante o dia & 1,09 & 1,00 & 1,062 & \multirow[t]{3}{*}{0,0000} \\
\hline Sono noturno & 1,23 & 1,00 & 1,066 & \\
\hline Usa medicamentos para dormir & 1,10 & 0,00 & 1,345 & \\
\hline \multicolumn{5}{|l|}{ 8. Lazer e recreação } \\
\hline Grupos de convivência & 2,52 & 3,00 & 0,879 & \multirow[t]{3}{*}{0,0000} \\
\hline No domicílio & 1,76 & 2,00 & 1,223 & \\
\hline Passeios & 2,39 & 3,00 & 0,957 & \\
\hline \multicolumn{5}{|l|}{ 9. Expressão da sexualidade } \\
\hline Exercita sua sexualidade & 2,80 & 3,00 & 0,671 & \multirow[t]{2}{*}{0,2810} \\
\hline Expressa sua sexualidade & 2,69 & 3,00 & 0,807 & \\
\hline \multicolumn{5}{|l|}{ 10. Comunicação } \\
\hline Compreensão cognitiva & 1,12 & 1,00 & 1,108 & \multirow[t]{4}{*}{0,000} \\
\hline Compreensão visual & 1,54 & 1,00 & 1,179 & \\
\hline Identifica sons & 0,91 & 0,00 & 1,098 & \\
\hline Verbaliza suas necessidades & 0,75 & 0,00 & 1,068 & \\
\hline \multicolumn{5}{|l|}{ 11. Uso de transportes } \\
\hline Sobe e desce dos transportes & 2,38 & 3,00 & 0,996 & \multirow[t]{3}{*}{0,0220} \\
\hline Transporte interurbano & 2,54 & 3,00 & 0,835 & \\
\hline Transportes urbanos & 2,54 & 3,00 & 0,828 & \\
\hline
\end{tabular}

